\title{
Thermal aging characterization of composite plates and honeycomb sandwiches by electromechanical measurement
}

\author{
Elhadji Barra Ndiaye, Hugues Duflo, Pierre Maréchal, ${ }^{a)}$ and Pascal Pareige \\ LOMC, UMR 6294 CNRS, Université du Havre, 75 rue Bellot, 76600 Le Havre, France
}

(Received 27 June 2017; revised 10 November 2017; accepted 27 November 2017; published online 20 December 2017)

\begin{abstract}
After identifying the parameters of the piezoelectric transducer, the mechanical impedance of the front medium is deduced. More particularly, the wave propagation velocity and attenuation are deduced in the inspected plate. By fitting the electrical impedance measurement results, the aging of composite materials is quantified, showing the effectiveness of this means of nondestructive evaluation. A specific measurement tool and protocol are proposed. Several estimators are identified on the basis of Gaussian fits of the resonances observed on the electrical impedance measurements. Those estimators are identified and the obtained results show that both the frequencies and widths of the resonances peaks vary according to the health of the plate, or aging duration. This method is applied successfully on non-perfect sandwich plates, porous, and with non-ideally flat surface. The sensitivity and limits of the most relevant estimators are discussed for the two studied plate families. (C) 2017 Acoustical Society of America. https://doi.org/10.1121/1.5017609
\end{abstract}

[JFL]

Pages: 3691-3702

\section{INTRODUCTION}

In this paper, an experimental method of characterization of aged composite materials is investigated. These composite materials are nowadays widely used in aeronautics, due to their good mechanical performance, both in terms of specific rigidity, and fatigue resistance compared to conventional metallic materials. However, these composites undergo both thermal and mechanical aging during their life cycle. We will study thermal aging at a thermostated temperature with increasing duration of aging. The electromechanical impedance measurement of a piezoelectric transducer in contact gives information on the medium in front of the transducer. ${ }^{1}$ When in contact with a composite plate, ${ }^{2-5}$ it makes it possible to evaluate aging by measurement of the proportion of air voids. Indeed, the presence of porosities in material causes greater attenuation of the ultrasonic waves. For a given plate, the ultrasonic estimates of wave velocity and attenuation can be related to the air void content. These ultrasonic parameters will thus constitute good estimators of the state of aging of composite materials. ${ }^{4,5}$ Thermo-oxidizing aging describes the state of degradation of composite materials during their life cycle. It induces notable changes to the aspect, to the color, to micrography, and to the thickness of materials. Preceding studies undertaken by Ammar-Khodja et $a l^{4}$ on the same samples reveal states of damage such as the appearance of cracks due to oxidation by the air after $1000 \mathrm{~h}$ of aging. ${ }^{5}$ With the increase in the duration of aging, the number and the depth of cracks increase but no degradation is detectable in the middle of the material. As a solution, the thickness vibration of piezoelectric disk is affected by the effective properties of the medium which is in contact. Thus, hydrolytic degradation of a composite was characterized through electromechanical impedance measurement. ${ }^{6-12}$ Thickness mode

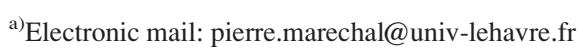

transduction, such as described by Mason's model, ${ }^{13-15}$ can be sufficient to relate aging to electroacoustic response. This method was also applied to very dispersive polymeric materials by Perrisin-Fabert and Jayet. ${ }^{6}$ The use of piezoelectric disks in low frequency, which corresponds to the radial modes, made it possible to detect defects such as delaminations. $^{16-18}$ Thus, Giurgutu and Zagrai ${ }^{8}$ and Giurgutu and Rogers ${ }^{7}$ showed the damage to composite beams, the detection of cracks in aluminum plates, the aging by detecting corroded zones of metal panels as well as other more complex structures. Other studies on the electromechanical impedance of piezoelectric patches have revealed levels of damage in structures made out of reinforced concrete and more generally in composite material structures. ${ }^{19-21}$ This aging is realized in an oxygen-depleted atmosphere in order to reduce the effects of oxidation by the air.

\section{THERMO-OXIDIZED COMPOSITE PLATES}

\section{A. Composite plates}

The composite plates were $2.3 \mathrm{~mm}$ thick, $300 \mathrm{~mm}$ length, and $200 \mathrm{~mm}$ width. They were made of eight woven folds of carbon fiber, in the form of a pre-impregnated epoxy matrix. During stacking, a sequence was strictly respected to obtain the mechanical performances of the material. The sequence of stacking $\left(\left[0^{\circ},+45^{\circ},-45^{\circ}, 90^{\circ}\right]_{\mathrm{s}}\right)$ was used before the operation of taping which consists of placing the folds on an aluminum plate and covering them by a whole of fabrics intended for the pumping of the resin surplus. Then, a vacuum cover was placed on the top of the tools to inhibit the appearance of porosities in the parts.

\section{B. Thermo-oxidation aging}

A batch of six aged plates, from 500 to $5000 \mathrm{~h}$ at a thermostated temperature of $180^{\circ} \mathrm{C}$, was characterized by 
TABLE I. Duration of the thermal aging of the composite plates.

\begin{tabular}{lcccccc}
\hline \hline Plate name & F01 & F02 & F03 & F04 & F05 & F06 \\
\hline Duration $D(\mathrm{~h})$ & 500 & 1000 & 1500 & 2500 & 3500 & 5000 \\
\hline \hline
\end{tabular}

the electromechanical measurement method, based on the electrical impedance measurement of a piezoelectric transducer $^{1}$ in contact with such composite materials. ${ }^{2,3}$ Table I shows the duration of aging of six plates in order to describe aging under the same conditions of degradation. Before studying the electromechanical behavior of the transducer on the plates, a scanning electron microscope was used to visualize into the depth of the laminates (Fig. 1). As illustrated by Fig. 1(a), in an initial state, one observes neither porosity nor cracks in the material. The second micrographs shown Fig. 1(b), was produced at the time of the follow-up of aging of the plate. These images illustrate the state of damage in the time characterized by thermal aging in an atmosphere impoverished of oxygen. This same type of aging is applied to the sandwiched samples but at various thermostated temperatures to provide additional data to the studies already conducted at $180^{\circ} \mathrm{C}$ on the aging of composite plates.

\section{MODELING}

\section{A. Electromechanical impedance measurement}

As a characterization facility, the electromechanical impedance measurement of a piezoelectric transducer in contact with a composite material ${ }^{2,6,7}$ makes it possible to evaluate the porosity content, i.e., aging properties, of the material. Thus, the ultrasonic estimates of wave velocity and attenuation constitute good estimators on the state of aging of composite materials. ${ }^{8,9}$ Previous studies have shown that the electrical impedance of a piezoelectric patch is modified in an important way when it is in contact with a viscoelastic medium ${ }^{10}$ or composite. ${ }^{11,12}$ In this study, a piezoelectric transducer is used which is modeled later using a Mason's model ${ }^{13-15}$ of piezoelectric layer.

\section{B. Transducer}

In basic terms, a piezoelectric transducer consists of at least of three media: the piezoelectric layer, inserted between the back medium and the matching layers, themselves in contact with the front medium. All the components of the transducer are conceived so as to widen the bandwidth of the transducer and to decrease the echo duration. By using the Mason modeling of a piezoelectric layer, ${ }^{13-15}$ the transducer can thus be represented by the following three port diagram (Fig. 2).

The piezoelectric layer is modeled using Mason's model:

$$
\left(\begin{array}{c}
-F_{\text {back }} \\
-F_{2} \\
V
\end{array}\right)=\left[\begin{array}{lll}
Z_{11} & Z_{12} & Z_{13} \\
Z_{12} & Z_{11} & Z_{13} \\
Z_{13} & Z_{13} & Z_{33}
\end{array}\right] \cdot\left(\begin{array}{c}
v_{\text {back }} \\
-v_{2} \\
I
\end{array}\right)
$$

where $F_{\text {back }}$ and $F_{2}$ are the forces, $v_{\text {back }}$ and $v_{2}$ are the velocities at the back and front face of the piezoelectric layer, respectively; $V$ is the voltage, $I$ is the current, $Z_{i j}$ are the elements of the electromechanical impedance matrix.

Each of the components of the rear medium results in $-F_{\text {back }}=Z_{\text {back }} v_{\text {back }}$, where $Z_{\text {back }}$ the mechanical impedance of the back medium, and this makes it possible to model the behavior of all the components of the front layers, including the matching layers, as follows:

$$
\left(\begin{array}{c}
-F_{2} \\
-F_{\text {front }}
\end{array}\right)=\left[\begin{array}{ll}
Z_{11}^{c} & Z_{12}^{c} \\
Z_{12}^{c} & Z_{22}^{c}
\end{array}\right] \cdot\left(\begin{array}{c}
v_{2} \\
-v_{\text {front }}
\end{array}\right),
$$

where $F_{2}$ and $F_{\text {front }}$ are the forces, $v_{2}$ and $v_{\text {front }}$ are the velocities at the front face of the piezoelectric layer and at the front face of the transducer, respectively; $Z_{i j}^{c}$ are the elements of the mechanical impedance matrix corresponding to the front layers.

Now, while considering the contributions of the back medium and the matching layers, the relation is established:

$$
\left(\begin{array}{c}
-F_{\text {back }} \\
V
\end{array}\right)=\left[\begin{array}{ll}
A_{11} & A_{12} \\
A_{21} & A_{22}
\end{array}\right] \cdot\left(\begin{array}{c}
v_{\text {back }} \\
I
\end{array}\right),
$$

where $A_{i j}$ are the elements of the reduced electromechanical impedance matrix, (a)

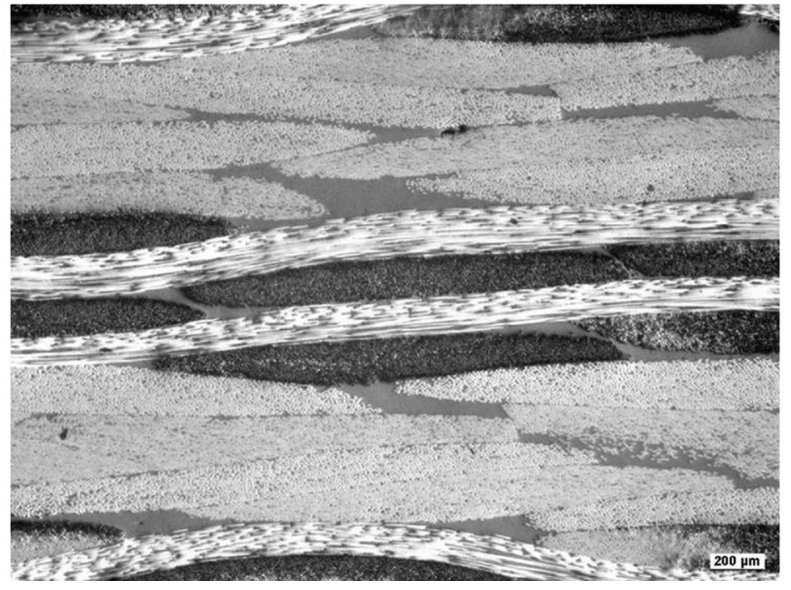

(b)

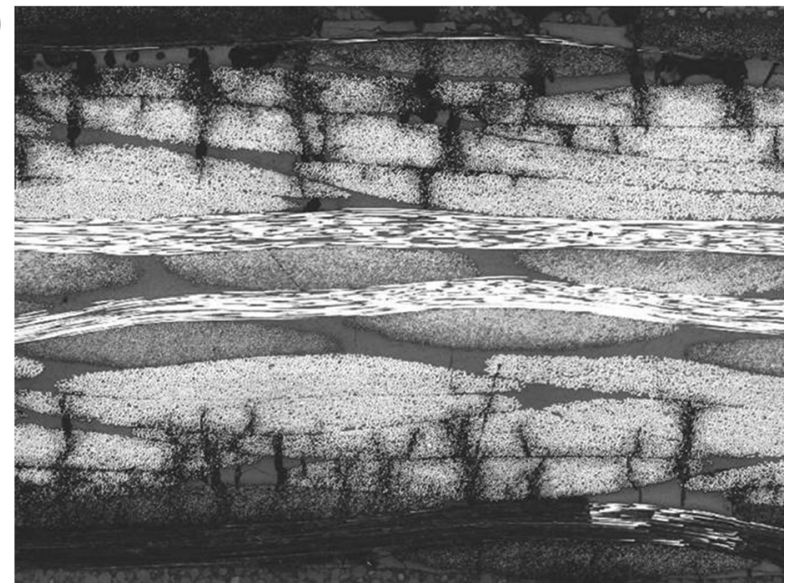

FIG. 1. Micrography of (a) a healthy plate and (b) $9000 \mathrm{~h}$ aged plate. 


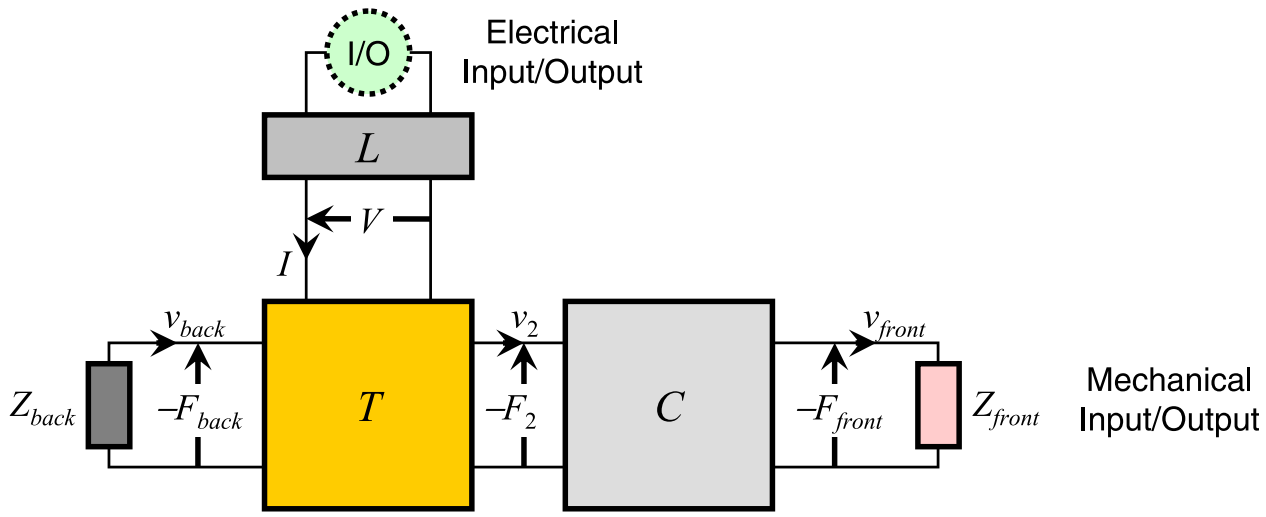

FIG. 2. (Color online) Three ports diagram of the equivalent system of the transducer with two couples of acoustical input/output parameters ( $F_{\text {back }}$, $\left.v_{\text {back }}\right),\left(F_{\text {front }}, v_{\text {front }}\right)$, and an electrical one $(V, I)$.

$$
\text { and }\left\{\begin{array}{l}
A_{11}=\frac{Z_{12}^{c} Z_{22}^{c}+\left(Z_{11}-Z_{\text {back }}\right)\left(\left(Z_{12}^{c}\right)^{2}-Z_{22}^{c}\left(Z_{11}+Z_{11}^{c}\right)\right)}{\left(Z_{11}^{c}+Z_{11}\right)\left(Z_{11}-Z_{\text {back }}\right)-\left(Z_{12}\right)^{2}} \\
A_{12}=\frac{Z_{13} Z_{12}^{c}\left(Z_{12}-Z_{11}+Z_{\text {back }}\right)}{\left(Z_{11}^{c}+Z_{11}\right)\left(Z_{11}-Z_{\text {back }}\right)-\left(Z_{12}\right)^{2}}=A_{21} \\
A_{22}=Z_{33}-\frac{\left(Z_{13}\right)^{2}}{Z_{11}-Z_{\text {back }}}\left(1-\frac{\left(Z_{12}\right)^{2}-\left(Z_{11}-Z_{\text {back }}\right)^{2}}{\left(Z_{11}^{c}+Z_{11}\right)\left(Z_{11}-Z_{\text {back }}\right)-\left(Z_{12}\right)^{2}}\right)
\end{array}\right.
$$

The piezoelectric transducer used an electromechanical converter is thus defined by the three elements of the reduced electromechanical matrix $A_{11}, A_{12}$, and $A_{22}$.

\section{Transducer coupled to various media}

The front face of the transducer is in contact with a medium of mechanical input impedance $Z_{\text {front }}$ (Fig. 2) whose expression can be written as

$$
Z_{\text {front }}=\frac{F_{\text {front }}}{v_{\text {front }}}=\frac{Z_{11}^{c} Z_{22}^{c}-\left(Z_{12}^{c}\right)^{2}}{Z_{11}^{c}} .
$$

As a result, the electrical impedance $Z_{T}$ seen from the electrical port of the transducer both depends on the set $\left\{A_{11}, A_{12}\right.$, $\left.A_{22}\right\}$ of electromechanical parameters and on the mechanical input impedance $Z_{\text {front }}$ seen by the front face of the transducer:

$$
Z_{T}=\frac{V}{I}=\frac{A_{22} Z_{\text {front }}-A_{11} A_{22}+A_{12}^{2}}{Z_{\text {front }}-A_{11}} .
$$

In order to determine the mechanical input impedance of the front medium $Z_{\text {front }}$ through the measurement of the electrical impedance of the transducer $Z_{T}$, it is an imperative to know precisely the set of electromechanical parameters $\left\{A_{11}, A_{12}, A_{22}\right\}$ which are characteristic of the transducer, as it will be described in the following.

\section{TRANSDUCER INVESTIGATION}

In order to determine properties such as the longitudinal wave velocity, attenuation and elastic constants in an elastic layer, it is possible to measure the electromechanical impedance of a transducer coupled to the same layer. This is a fast and quasi punctual method since the active surface of the transducer is in contact with the part to be characterized. A coupling gel ensures the transmission of the ultrasonic waves that are substantially planar in the propagation medium. This method can also be used to study the aging of multilayer environments such as composites or sandwiched materials.

\section{A. Measurements}

The measurement setup is shown in Fig. 3(a). A device consisting of propagating lines (with characteristic impedance $Z_{0}=50 \Omega$ and velocity of propagation $v_{0} \leq c_{0}=3 \times 10^{8} \mathrm{~m} / \mathrm{s}$ ) and a voltage divider is used to perform measurements of impedance of the $\mathrm{I} / \mathrm{O}$ device in the frequency range of

(a)



(b)

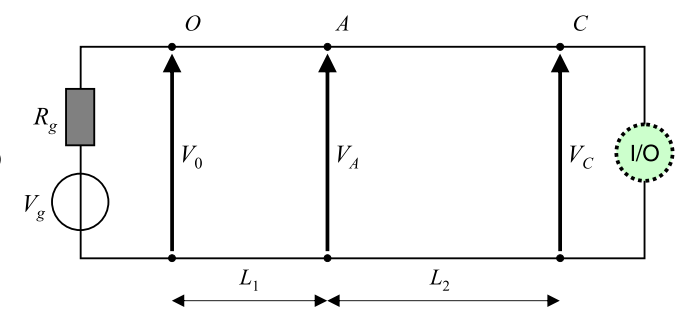

FIG. 3. (Color online) Measurement device: (a) experimental setup and (b) electrical equivalent scheme. 
1-100 MHz. The electrical equivalent scheme illustrated in Fig. 3(b) shows a low frequency generator (LFG) sending a signal $V_{0}$ to the point $A$ through the line $L_{1}$. To the emitting signal $V_{\text {emi }}$ is added the received one $V_{\text {refl }}$ after reflection on the electrical port of the transducer through the line $L_{2}$. At the point $A$, the probe permits the two waves $V_{\text {emi }}$ and $V_{\text {refl }}$ to be measured as

$$
\left\{\begin{array}{l}
V_{\mathrm{emi}}=V_{0} e^{-j k_{0} L_{1}} \\
V_{\mathrm{refl}}=V_{\mathrm{emi}} R e^{-j k_{0} 2 L_{2}} .
\end{array}\right.
$$

By defining the reflection coefficient $R$ as

$$
R=\frac{Z_{T}-Z_{0}}{Z_{T}+Z_{0}},
$$

we can express the resulting difference of voltage $V_{A}=V_{\mathrm{emi}}$ $+V_{\text {refl }}$ in the point $\mathrm{A}$ as

$$
V_{A}=V_{\mathrm{emi}}\left(1+R e^{-j k_{0} 2 L_{2}}\right)
$$

Equation (9) can be rewritten using the ratio $Q=V_{A} / V_{\text {emi }}$ as follows:

$$
R=(Q-1) e^{j k_{0} 2 L_{2}} .
$$

The impedance $Z_{T}$ of a transducer connected at the end of the line $L_{2}$ is deduced from the relation defined in Eq. (8). By replacing the reflection coefficient $R$ with the relation found in Eq. (10), the impedance $Z_{T}$ can be written as

$$
Z_{T}=Z_{0} \frac{1+(Q-1) e^{j k_{0} 2 L_{2}}}{1-(Q-1) e^{j k_{0} 2 L_{2}}} .
$$

Now when the transducer is coupled to any medium (i.e., any devices at the end of the line $L_{2}$ ), the impedance $Z_{T}$ tends to infinity and therefore the reflection coefficient $R=1$. Thus the difference of voltage $V_{C}=V_{\text {refl }}($ with $R=1)$ :

$$
V_{C}=V_{\mathrm{emi}}\left(1+e^{-j k_{0} 2 L_{2}}\right) \text {. }
$$

We can also define the ratio $P=V_{C} / V_{\text {emi }}$, and finally the impedance of a transducer connected at the end of the line $L_{2}$ is expressed as

$$
Z_{T}=Z_{0} \frac{1+\frac{Q-1}{P-1}}{1-\frac{Q-1}{P-1}}=Z_{0} \frac{P+Q-2}{P-Q} .
$$

The medium to be characterized acoustically is set in contact with the front face of the transducer and measurements of the signals $V_{\text {emi }}, V_{B}$, and $V_{C}$ are being conducted (Fig. 3). The spectral transfer functions $P$ and $Q$ are then determined by fast Fourier transform (FFT), and the electrical impedance of the transducer $Z_{T}$ [Eq. (13)] is then calculated, expressed and illustrated in terms of modulus $\left|Z_{T}\right|$ [Fig. 4(a)] and phase $\Phi_{Z_{T}}$ [Fig. 4(b)], as a function of the frequency $f$, here in contact with air. The modeling and the experimental results are in good agreement.

\section{B. Identification of transducer parameters $A_{11}, A_{12}$, and $\boldsymbol{A}_{22}$}

The relation established in Eq. (5) permits us to model the electromechanical impedance of a transducer in contact with a layer. This is only possible through the knowledge of the parameters $A_{11}, A_{12}$, and $A_{22}$ in the frequency range use of the transducer. The identification of the three parameters is made possible by taking into account three known media having different impedances. In this way, measurements are conducted, respectively, with these three media: air, poly(methyl methacrylate) (PMMA), aluminum. As illustrated in Fig. 5, these three measurement results of the electrical impedance $Z_{T}$ of a $1 \mathrm{MHz}$ center frequency transducer are presented with its modulus $\left|Z_{T}\right|$ in Fig. 5(a) and its phase $\Phi_{Z_{T}}$ in Fig. 5(b). From these experimental results, the complex parameters $A_{11}, A_{12}$, and $A_{22}$ characterizing the transducer are identified. Then, we obtain a system of three equations with the three parameters. The numerical values used for the triplet of materials are summarized in Table II. The triplet of media \{air, PMMA, aluminum\} gives the results of the full set of complex parameters $\left\{A_{11}, A_{12}, A_{22}\right\}$. These electromechanical complex parameters $A_{11}, A_{12}$, and $A_{22}$ are plotted within the frequency bandwidth of the transducer following their real parts [Figs. 6(a), 6(c), 6(e)] and imaginary parts [Figs. 6(b), 6(d), 6(f)], respectively. Some other triplets of materials have been tested to verify the

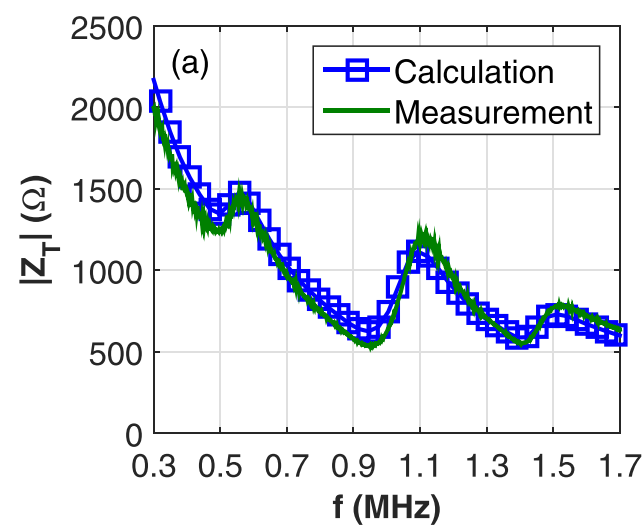

FIG. 4. (Color online) Comparison between the calculation and measurement of the electrical impedance $Z_{T}$ of the transducer while in contact with air: (a) modulus $\left|Z_{T}\right|$ and (b) phase $\Phi_{Z_{T}}$. 

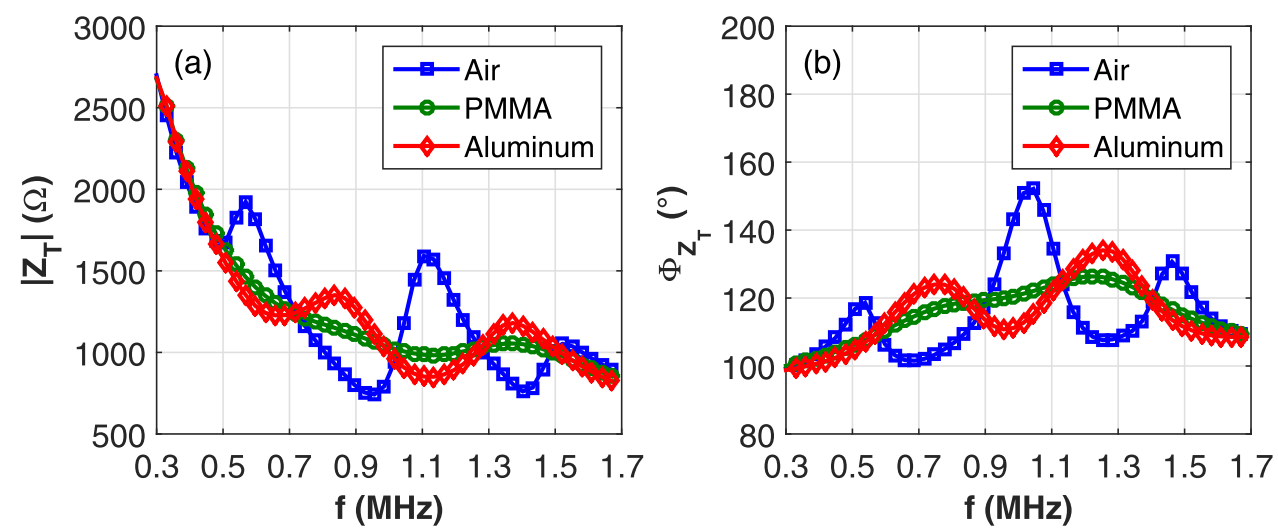

FIG. 5. (Color online) Comparison between the calculated electrical impedance $Z_{T}$ of the transducer coupled with air, coupled with PMMA and coupled with aluminum: (a) modulus $\left|Z_{T}\right|$ and (b) phase $\Phi_{Z_{T}}$.

accuracy of the identification, and these give the same results, what confirms the independence of the $A_{i j}$ parameters relatively to the set of reference materials, since they present a high acoustical impedance contrast. After determining the characteristics of the transducer, these parameters can be used to solve the inverse problem, i.e., the characterization of the front medium.

\section{Inverse calculus for extraction of mechanical characteristics}

The electrical impedance $Z_{T}$ of the transducer in contact with a medium provides some information. Thus, once the set of three electromechanical parameters of the transducer $\left\{A_{11}, A_{12}, A_{22}\right\}$ is known, the front mechanical impedance $Z_{\text {front }}$ is deduced by the measurement of the electrical impedance $Z_{T}$ of the transducer. Reciprocally to Eq. (5), the following relation can be established:

$$
Z_{\text {front }}=\frac{A_{11} Z_{T}-A_{11} A_{22}+A_{12}^{2}}{Z_{T}-A_{22}}
$$

On the other side, the mechanical impedance $Z_{\text {front }}$ corresponds to the elastic assembly layers and the propagating medium consisting of the coupling layer and the plate. Thus, it results in the following relation:

$$
Z_{\text {front }}=j Z_{c} \frac{Z_{p} \tan \left(\theta_{p}\right)+Z_{c} \tan \left(\theta_{c}\right)}{Z_{c}-Z_{p} \tan \left(\theta_{c}\right) \tan \left(\theta_{p}\right)}
$$

where $\left\{Z_{c}, \theta_{c}\right\}$ and $\left\{Z_{p}, \theta_{p}\right\}$ are the mechanical properties of the coupling layer (index $c$ ) and the plate (index $p$ ), respectively. The mechanical impedance is given by $Z=A \rho c$, with $A$ the area of the active surface, $\rho$ the density and $c$ the wave

TABLE II. Acoustical properties of the reference media. $\rho$ : density; $c$ : longitudinal velocity; $\alpha$ : longitudinal attenuation; $Z_{a c}$ : acoustical impedance.

\begin{tabular}{lcccc}
\hline \hline Material & $\rho\left(\mathrm{kg} / \mathrm{m}^{3}\right)$ & $c(\mathrm{~m} / \mathrm{s})$ & $\alpha$ & $Z_{a c}$ (MRay) \\
\hline Air & 1.29 & 340 & - & 0.004 \\
PMMA & 1180 & 2730 & 0.001 & 3.22 \\
Aluminum & 2700 & 6300 & 0.001 & 17.01 \\
\hline \hline
\end{tabular}

velocity of the medium of interest and the angular delay results from $\theta=k d=2 \pi f d / c=2 \pi f t$, where $t$ is the time of flight. We consider the composite plate elastic constants to be complex and the $C_{33}$ modulus along the axis of propagation becomes $C_{33}=C_{33_{\mathrm{Re}}}(1+j \alpha)$ and the wave velocity also becomes complex as $c_{L}=c_{L_{\mathrm{Re}}} \sqrt{1+j \alpha}$. The influence of the transducer is suppressed by using the relation (14). The propagating medium is also modeled by using the relation (15). Therefore, by fitting the model to the experimental data, three characteristic parameters can be identified: the time of flight $t_{p}=d_{p} / c_{p}$, the mechanical impedance $Z_{p}=A \rho_{p} c_{p}$ and the mechanical loss coefficient $\alpha_{p}$. To assess the quality of the agreement between the theoretical model and experimental results, it is necessary to minimize the distance $\Delta Y$ between $Y_{E}$ the experimental electrical admittance and $Y_{T}$ the theoretical electrical admittance calculated using the model at the same frequencies:

$$
\begin{aligned}
\Delta Y\left(Y_{T}, Y_{E}\right)= & \frac{1}{N_{f}} \sum_{n=1}^{N_{f}}\left|\Re e\left(Y_{E, n}-Y_{T, n}\right)\right| \\
& +\left|\Im m\left(Y_{E, n}-Y_{T, n}\right)\right|,
\end{aligned}
$$

where $\Delta Y\left(Y_{T}, Y_{E}\right)$ is the a distance estimate which is calculated as the average on $N_{f}$ frequency samples of the absolute difference of the real and imaginary parts of the experimental $Y_{E, n}$ and theoretical $Y_{T, n}$ electrical admittance sampled data. This distance estimate $\Delta Y\left(Y_{T}, Y_{E}\right)$ between theory and experiment gives a significant weight to the peak position which is a kind of acoustical signature of the plate. Indeed, a slight frequency shift of a peak causes a significant variation in the distance estimate $\Delta Y\left(Y_{T}, Y_{E}\right)$. As a result, this broadband electrical impedance measurement gives the physical properties of an aged plate. Therefore, several parameters are deduced from the inverse problem and plotted as a function of the aging duration: the propagation delay $t_{p}$ [Fig. 7(a)], mechanical impedance $Z_{p}$ [Fig. 7(b)], longitudinal attenuation $\alpha_{p}$ [Fig. 7(c)] and longitudinal velocity $c_{p}$ [Fig. $\left.7(\mathrm{~d})\right]$ are showing some significant variations enabling to estimate the aging of the plates. In Sec. V we investigate an efficient, i.e., strongly correlated and less time-consuming characterization method by evaluating widths and peak positions of the resonance spectra. 

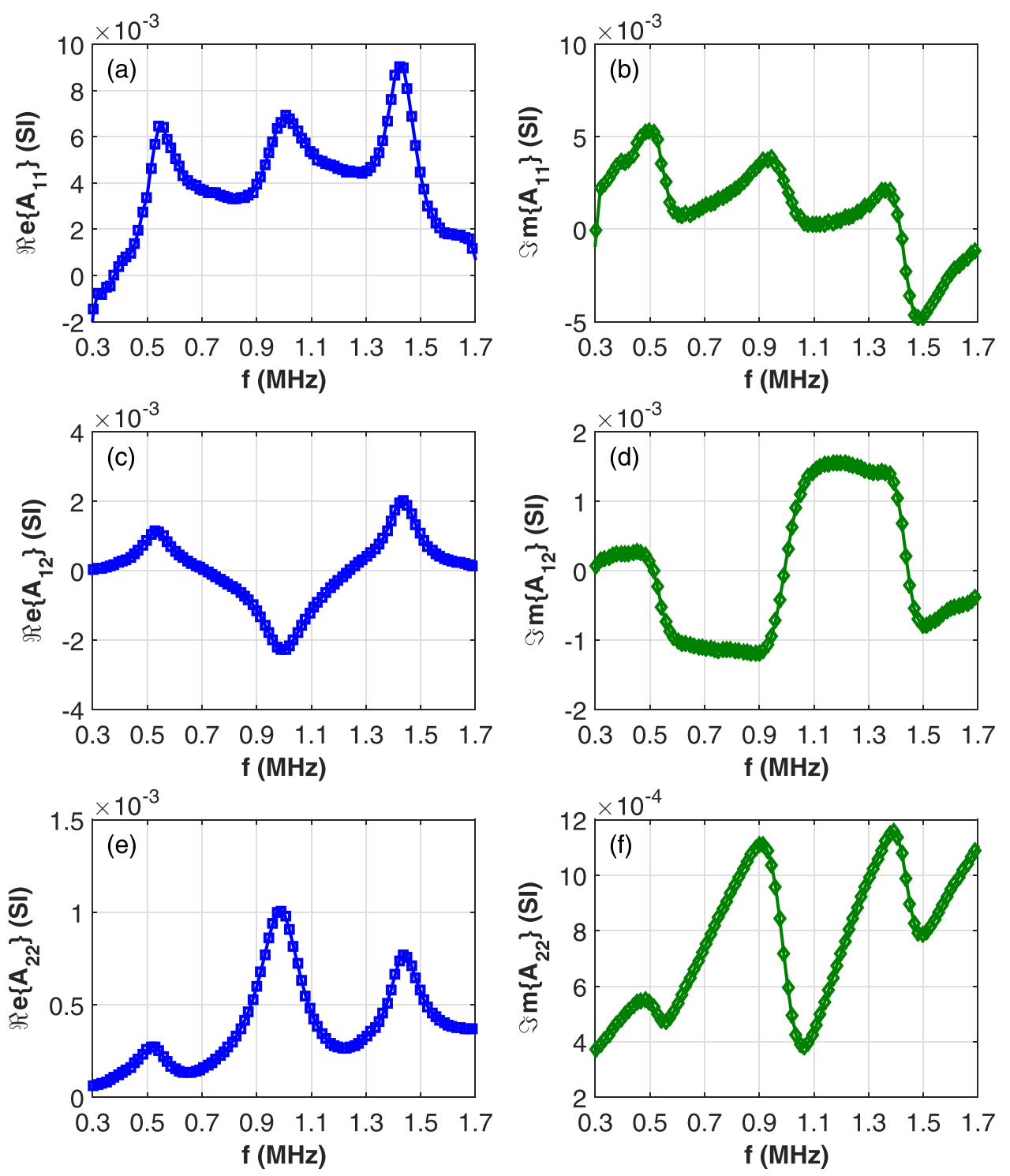

FIG. 6. (Color online) Variations of the Mason's $A_{i j}$ transducer parameters according to the frequency: (a), (c), (e) real part $\Re \mathrm{e}\left\{A_{i j}\right\}$ and (b), (d), (f) imaginary part $\mathfrak{J} \mathrm{m}\left\{A_{i j}\right\}$.
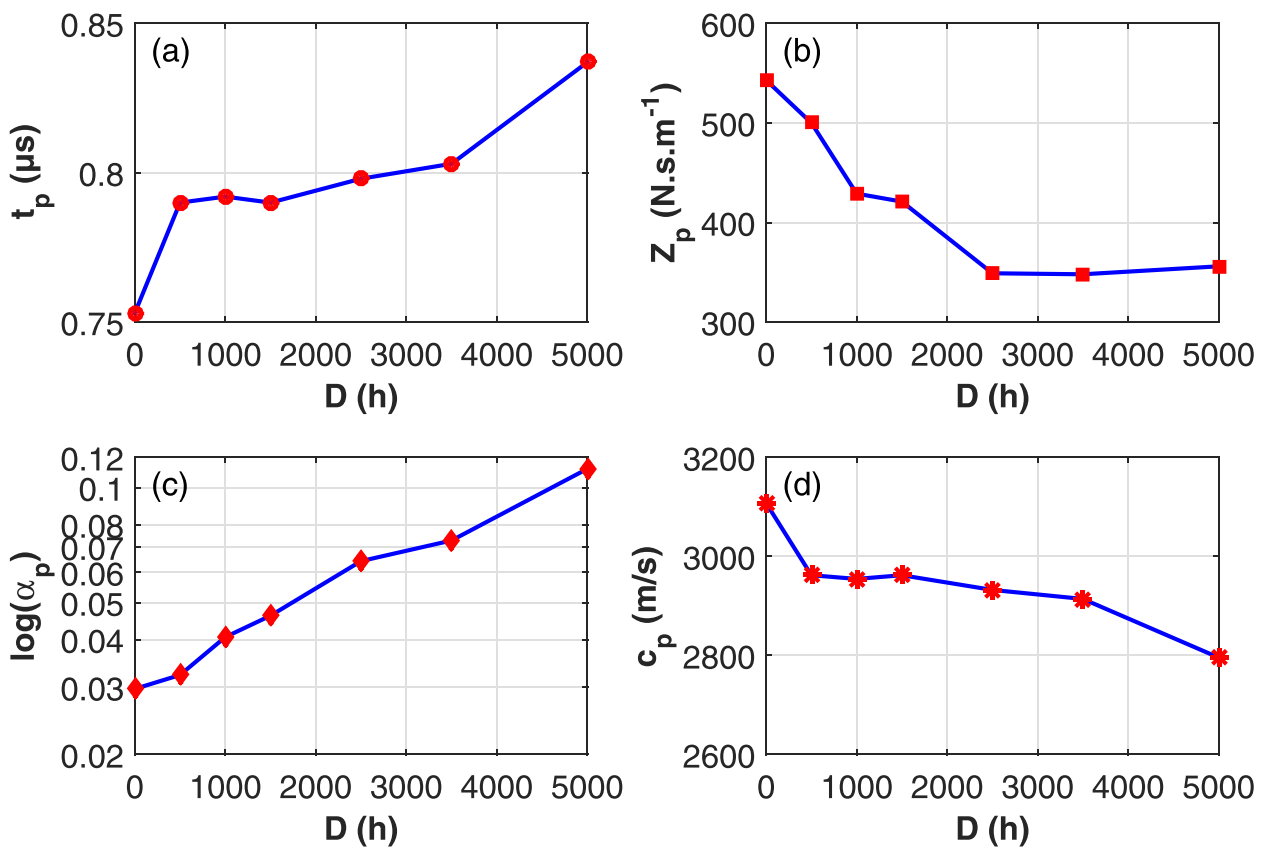

FIG. 7. (Color online) Evolution of characteristic parameters of the plate as a function of aging time: (a) propagation time $t_{p}(\mathrm{~s})$, (b) mechanical impedance $Z_{p}\left(\mathrm{~N} \mathrm{~s} \mathrm{~m}^{-1}\right)$, (c) attenuation in $\operatorname{logarithmic}$ scale $\log \left(\alpha_{p}\right)$ and (d) velocity $c_{p}(\mathrm{~m} / \mathrm{s})$. 


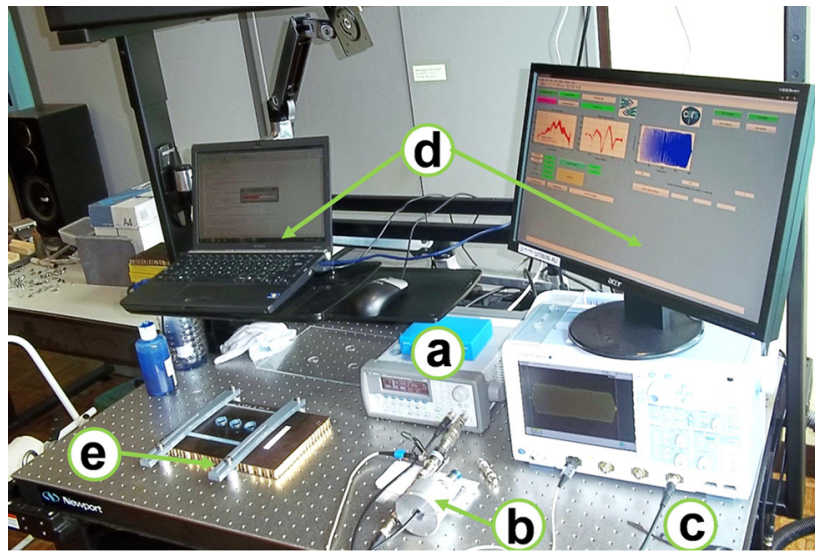

FIG. 8. (Color online) Experimental setup: (a) signal generator (Agilent $33220 \mathrm{~A}$ ), (b) calibrated pressure device of the contact transducer on the plate, (c) oscilloscope (Yokogawa DL9140), (d) Matlab specifically developed software and (e) fixing transparent plate device with positioning holes.

\section{DETERMINATION OF THE NEW CRITERIA}

\section{A. Experimental device}

For this study of the characterization of thermal aging, specific measuring equipment has been developed. It consists of a $1 \mathrm{MHz}$ central frequency broadband transducer fixed on a tripod. An acoustical gel ensures the coupling between the plate and the transducer. The tripod allows right angle positioning of the transducer on the composite sample. The tripod which receives the transducer is equipped with a spring system creating a constant pressure of the contact between the sensor and the composite plate. A signal generator (Agilent 33220A) allows the generation of a windowed chirp. A specific software has been developed for these experiments. It allows data acquisition by means of an oscilloscope (Yokogawa DL9140), signal processing, spectral analysis and also a graphical presentation of results (Fig. 8). The diameter of the transducer is just slightly larger than a cell of the honeycomb. To realize the measurements, a transparent plate was used with positioning holes having the same diameter as the transducer. This allows us to make measurements always at the same place on the composite. Moreover, holes are made so that the sensor is exactly located with regard to the center of a cell of the honeycomb.

\section{B. Calibration and characteristics of the chirp}

To create the necessary vibrations for the measurements of electromechanical impedance, we generate an incident chirp signal. A chirp is defined by its duration $\delta$ and frequency range $\left(f_{2}-f_{1}\right)$, i.e., a frequency modulated signal with variations from a low frequency $f_{1}$ to a higher frequency $f_{2}$. The general expression of a linear chirp with $f(t)=f_{1}+\left(f_{2}\right.$ $\left.-f_{1}\right) / \delta t$ can be established as follows:

$$
s(t)=A \sin \left(2 \pi\left(f_{1}+\frac{\left(f_{2}-f_{1}\right) t}{2 \delta}\right) t\right),
$$

with $\delta=200 \mu$ s the duration of the chirp, $f_{1}=0.2 \mathrm{MHz}$ and $f_{2}=1.8 \mathrm{MHz}$, respectively, the starting and ending frequencies, and $A=10 \mathrm{~V}$ the voltage amplitude. This chirp is windowed by a weighting function $w(t)$ to guarantee smooth transitions, with a rise time $t_{r}$ and a decay time $t_{d}$. The rise time is defined by the time required to pass from $10 \%$ to $90 \%$ (reversely the decay time) of its value in the established regime. Using a half cosine function both for the rise and decay of the windowing function $w(t)$, with a half period $T_{r}=T_{d}=15 \mu \mathrm{s}$, it results in a rise time and a decay time $t_{r}=t_{d}=8.83 \mu \mathrm{s}$. Therefore, the applied voltage is $U(t)$ $=s(t) w(t)$, these characteristics are shown in Fig. 9(a) and its corresponding spectrum modulus $U_{f}(f)$ is illustrated in Fig. 9(b). The resulting half height bandwidth $B W_{6}$ is slightly reduced from 0.26 to $1.74 \mathrm{MHz}$ instead of the range from $f_{1}=0.2 \mathrm{MHz}$ to $f_{2}=1.8 \mathrm{MHz}$, due to the windowing effect. Nevertheless, the excitation frequency range is wide enough, i.e., wider than the transducer's bandwidth.

\section{Acquisitions and tests}

All the studied plates were aged in a thermo-oxidizing atmosphere at a constant temperature of $180^{\circ} \mathrm{C}$. The thermooxidizing aging was chosen because it reflects the aging conditions in the ambient air of a composite material used in an aeronautical structure. The temperature and the oxidation both contribute to the aging. During the thermal aging, the air contained in the oven is constantly renewed. This prevents any saturation by all kinds of chemicals that would modify the process of aging. The aim of this protocol is to identify the measurement chain. It must be repeated after any modification of the experimental set up. Experiments are performed using a three-step approach. First the chirp signal is sent to the transducer without any contact (transducer loading acquisition "TLA"), then to a $50 \Omega$ resistance (resistance loading acquisition "RLA"). Finally, the experiment is done with the transducer in contact with the sample to be characterized (test acquisition "TA"). The analysis sample is
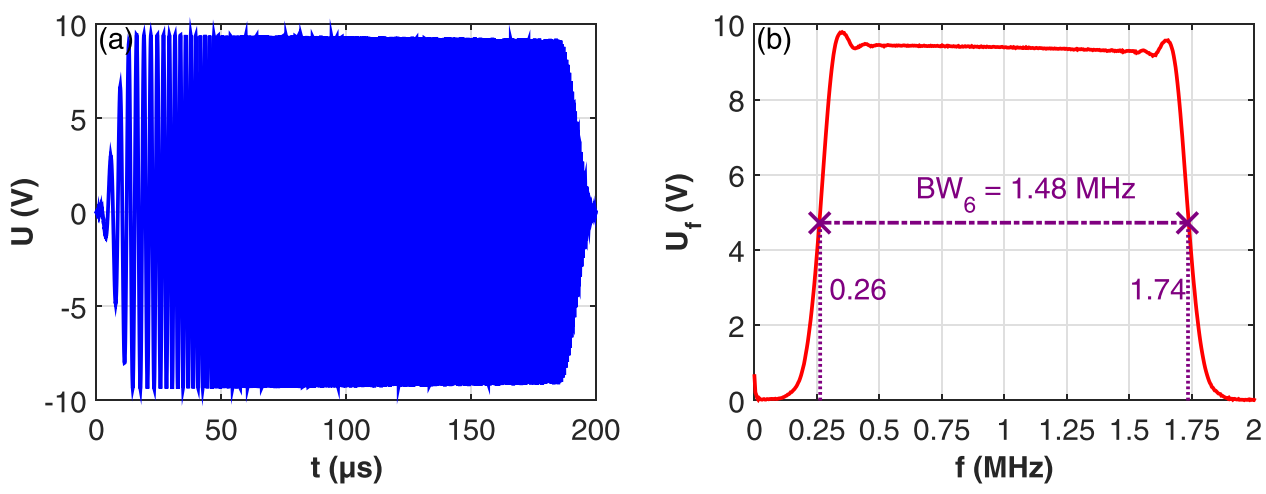

FIG. 9. (Color online) Electrical excitation signal: linear chirp with (a) a time waveform with a cosine windows duration $\delta=200 \mu$ s including rise and decay times $t_{r}=t_{d}=8.83 \mu \mathrm{s}$, (b) spectrum ranging from $f_{1}=0.2$ to $f_{2}=1.8$ MHz. 

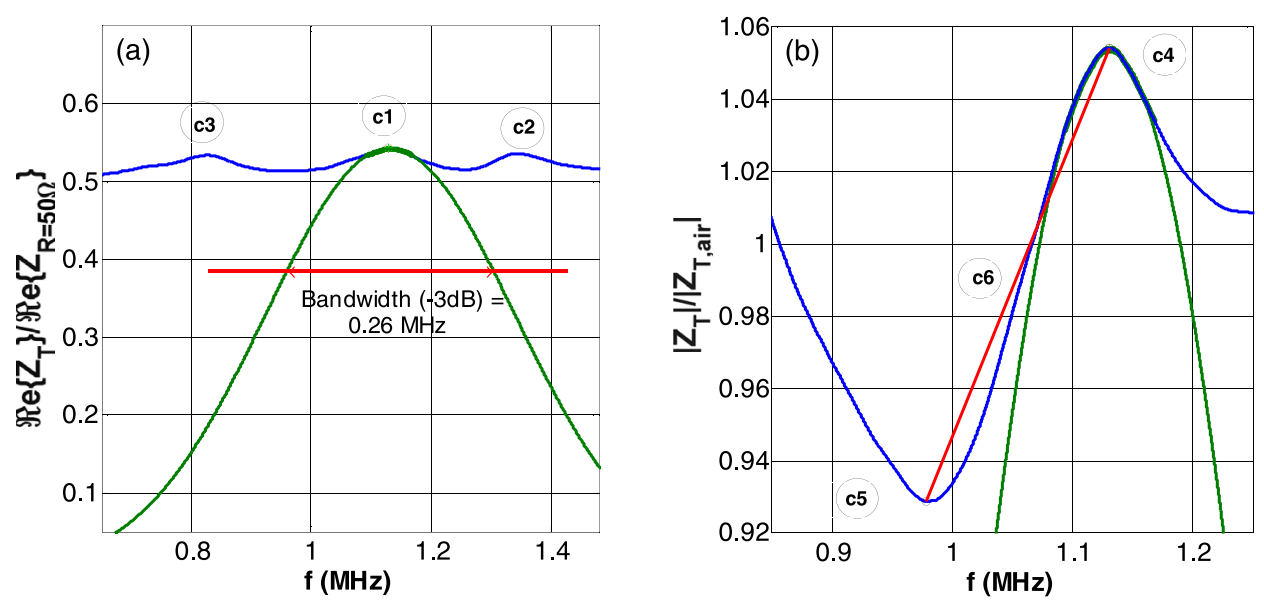

FIG. 10. (Color online) Gaussian $-3 \mathrm{~dB}$ bandwidths and slope criteria: (a) bandwidths $\mathrm{c} 1, \mathrm{c} 2$, and $\mathrm{c} 3$ defined from fits on the ratio of the real parts of the spectra TA/RLA denoted as $\Re \mathrm{e}\left\{Z_{T}\right\} /$ $\Re \mathrm{e}\left\{Z_{R=50 \Omega}\right\}$. (b) Bandwidths c4, c5, and slope $\mathrm{c} 6$ defined from fits on the ratio of the modules of the spectra TA/ TLA denoted as $\left|Z_{T}\right| /\left|Z_{T \text {,air }}\right|$. here a monolithic composite plate having suffered a thermooxidizing aging. The spectrum of these three temporal signals is obtained by FFT and is analyzed within the bandwidth of the transducer, i.e., from 0.5 to $1.5 \mathrm{MHz}$. These raw data are used to propose estimators based on a reproducible characterization method. As a result, a normalization is carried out with the ratio of the real parts of the complex frequency signals TA/RLA (curve 1) as illustrated in Fig. 10(a) and denoted as $\Re \mathrm{e}\left(Z_{T}\right) / \Re \mathrm{e}\left(Z_{R}=50 \Omega\right)$. An alternative way of normalization consists in the ratio of the modules of the complex frequency signals TA/TLA (curve 2) as illustrated in Fig. 10(b) and denoted as $\left|Z_{T}\right| /\left|Z_{\text {alone }}\right|$. This work is first done for a reference plate which is a healthy plate without thermal aging, and then for a plate suffering thermal degradation. In both cases, two curves are obtained and our characteristic parameters can be identified. The proposed estimators are based on Gaussian function fits on each peak of the normalized electrical impedance. The local maxima are thus normalized in amplitude and fitted on a bandwidth of $\pm 35 \mathrm{kHz}$, and the bandwidth at $-3 \mathrm{~dB}$ of each peak can be determined. Figure 10(a) illustrates the calculation and the position of the criterion 1 denoted $\mathrm{c} 1$ which is situated near the $1 \mathrm{MHz}$ central frequency of the transducer. Similarly, this was also done for peaks referenced as c2 and c3. In Fig. 10(b) is illustrated the criterion c6 consisting in the value of the slope between the extreme values $c 4$ and $c 5$. In short, criteria $\mathrm{c} 1$ to $\mathrm{c} 5$ are defined as the $-3 \mathrm{~dB}$ bandwidth of the normalized Gaussian fit and c6 is the slope between the extreme values corresponding to $\mathrm{c} 4$ and $\mathrm{c} 5$. The greater the difference between the values of the criteria obtained for the plate to be tested and the values of the criteria obtained for the healthy reference plate, the more important is the degradation of the material to be tested. Afterward this observation, all the presented results will be normalized by those obtained for the composite sample F01. The plate F01 was thermally aged during the shortest period $(500 \mathrm{~h})$.

\section{Results}

The aging is estimated by means of the values of the criteria previously described (widths of peaks and a slope). To guarantee the validity of the results, we make at least ten measures for the same point. Between each test the transducer is completely decoupled from the plate and repositioned at the same place. For every criterion we calculate its mean value and the standard deviation stemming from the four measures. To obtain the widths of peaks values, we need to determine Gaussian curves associated with the maxima of the spectra. The detection of these maxima and the calculation of the Gaussian curves are made by a specifically developed software as illustrated in Fig. 10 by the localization of the criteria and in Fig. 11 by examples of numerical values. On these figures, we only see the peak and its \pm 35 $\mathrm{kHz}$ frequency neighborhood used for the calculated Gaussian curve. The parameter which is of interest is the bandwidth $\Delta f(\mathrm{~Hz})$ of the Gaussian at $-3 \mathrm{~dB}$. To obtain the value of the slope, we need to identify the extrema of the Gaussian curves which correspond to the main peak and the main hollow of each spectrum. The slope of the straight line connecting these extrema is the last criterion. On the curve
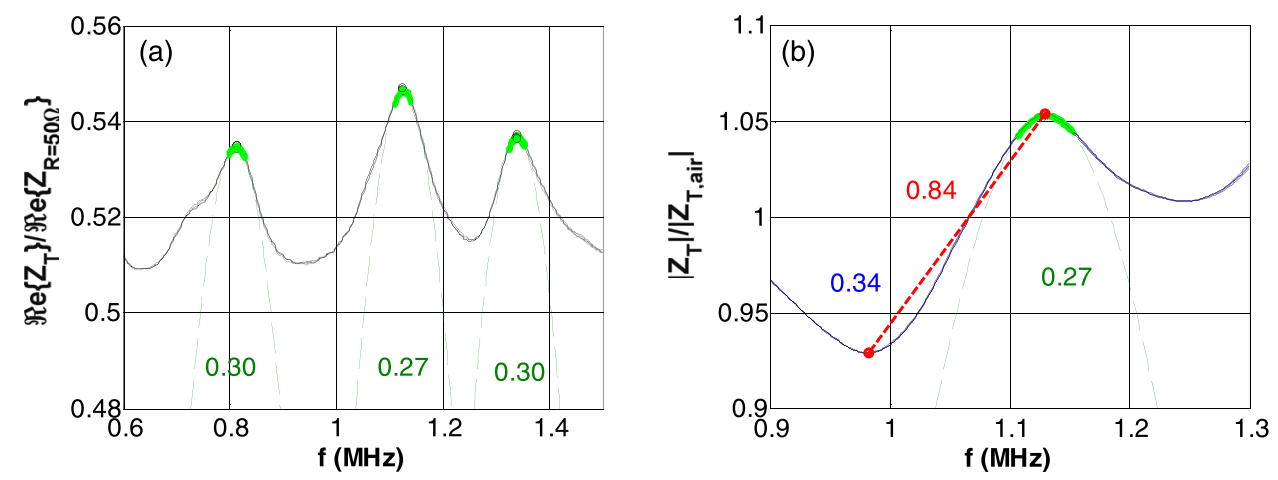

FIG. 11. (Color online) Determination of the positions and Gaussian fits of peaks on the reference plate F01 leading to the criteria values of (a) bandwidths c1, c2, c3, and (b) bandwidths c4, c5, and slope c6. 

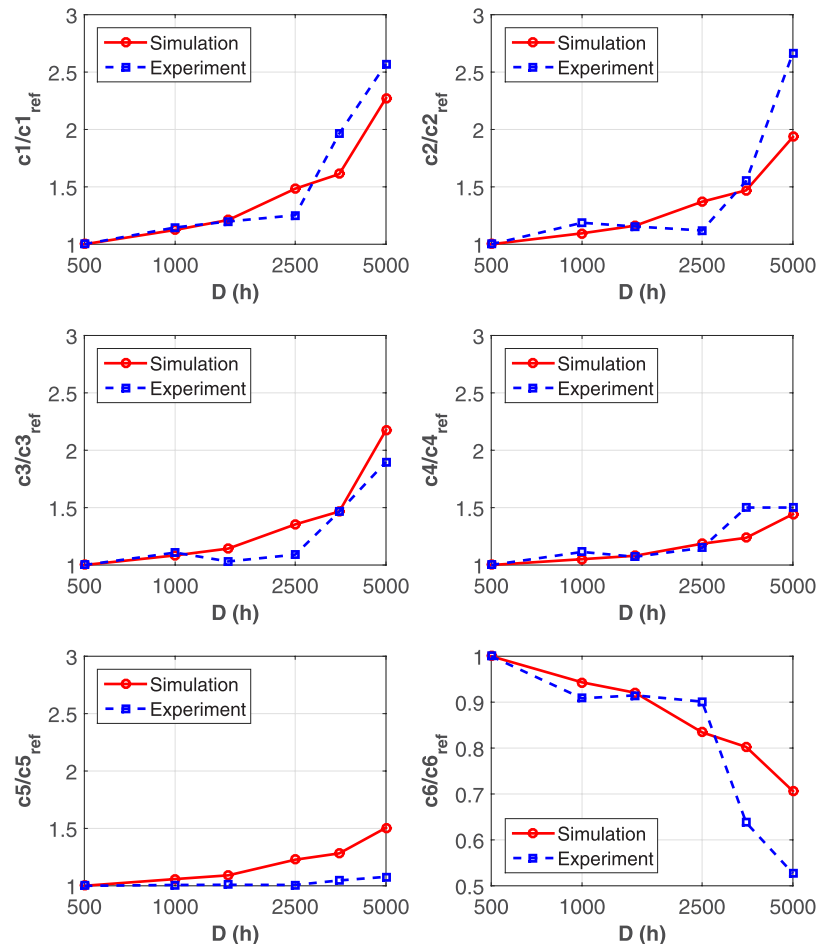

FIG. 12. (Color online) Comparison between simulation (red circles) and experiment (blue squares) with the evolution of the criteria (a) $\mathrm{c} 1 / \mathrm{c} 1_{\text {ref }}$, (b) $\mathrm{c} 2 / \mathrm{c} 2_{\text {ref }}$, (c) $\mathrm{c} 3 / \mathrm{c} 3_{\text {ref }}$, (d) $\mathrm{c} 4 / \mathrm{c} 4_{\text {ref }}$, (e) $c 5 / \mathrm{c} 5_{\text {ref }}$, (f) $c 6 / c 6_{\text {ref }}$, according to the aging duration for the monolithic plates.

which illustrates this paragraph the slope is drawn in red. It is a dimensionless value. The experimental process is repeated for every plate of series $\mathrm{F}$ aged for 500, 1000, 1500, 2500, 3500, and $5000 \mathrm{~h}$, respectively. In Figs. 12(a)-12(f), the evolution of the normalized criteria $\mathrm{c} 1 / \mathrm{c} 1_{\text {ref }}$ to $c 6 / c 6_{\text {ref }}$ is plotted according to the duration of aging, respectively. For criteria 1-5, the relative evolution of the bandwidths in $\mathrm{MHz}$ $(\Delta f)$ is shown. For the last one, criterion 6 , the slope of the straight line is given in $\mathrm{MHz}^{-1}\left(\Delta f^{-1}\right)$. In all the cases, it can noticed that for a thermal aging duration less than $2500 \mathrm{~h}$, the values of the bandwidths are slightly increasing and the slope is slightly decreasing. Thus, the variations of the values on the dot graph are more marked. Excepted for criterion
5, the errors bars linked to the standard deviation are all very small. Excepted for criterion 4, the values vary continuously with the duration of aging. This may be explained by the rather small range of variation for these bandwidths (30 and $100 \mathrm{kHz}$ for criteria 5 and 4, respectively). Globally, we note that the criteria are sensitive to the thermal aging of plates. It also seems that, beyond $2500 \mathrm{~h}$ of thermal aging, the appearance of local damage inside the plates accelerates, and then increases the variation of the criteria. To confirm these first results, a model of the thermal aging of the plates was carried out using their mechanical characteristics (Fig. 7) and those of the transducer (Fig. 6). With this model and by taking into account the evolution of the impedance $Z_{p}$, the time of flight $t_{p}$, the attenuation $\alpha_{p}$ and the longitudinal velocity $V_{p}$, we can recalculate the values of the criteria according to the duration of aging. For the best comparison of the results obtained by the measures and the modeling, all the values have been normalized with regard to those of the first plate (F01). Plate F01 which has the shortest thermal aging time is taken as a reference. In these conditions, Fig. 12 exhibits the normalized values of the criteria $\mathrm{c} 1$ to $\mathrm{c} 6$, by using the identified characteristics of the aged plates (simulation in red) and the values obtained experimentally (experiment in blue). We can notice a general good agreement except for criteria 4 and 5 , as mentioned previously.

\section{APPLICATION TO SANDWICH HONEYCOMB PLATES}

\section{A. Configuration}

Sandwich materials, with skins of carbon fiber reinforced plastics (CRFP) with bismaleimide (BMI) are shaped together in autoclave at a constant pressure. These sandwiches structures [Fig. 13(a)] are constituted of several functional layers, with a $1.6 \mathrm{~mm}$ thick skin made of 3 plies of CRFP/BMI and 1 ply of glass fiber, an adhesive layer for the $10 \mathrm{~mm}$ thick aluminum honeycomb grid core with $3 / 8$ in. cells, and symmetrically on the other side. The core of these sandwiches is an aluminum honeycomb that provides both lightness and stiffness to these materials. Thermal aging at two different temperatures $\left(170^{\circ}\right.$ and $\left.200^{\circ} \mathrm{C}\right)$ is applied with
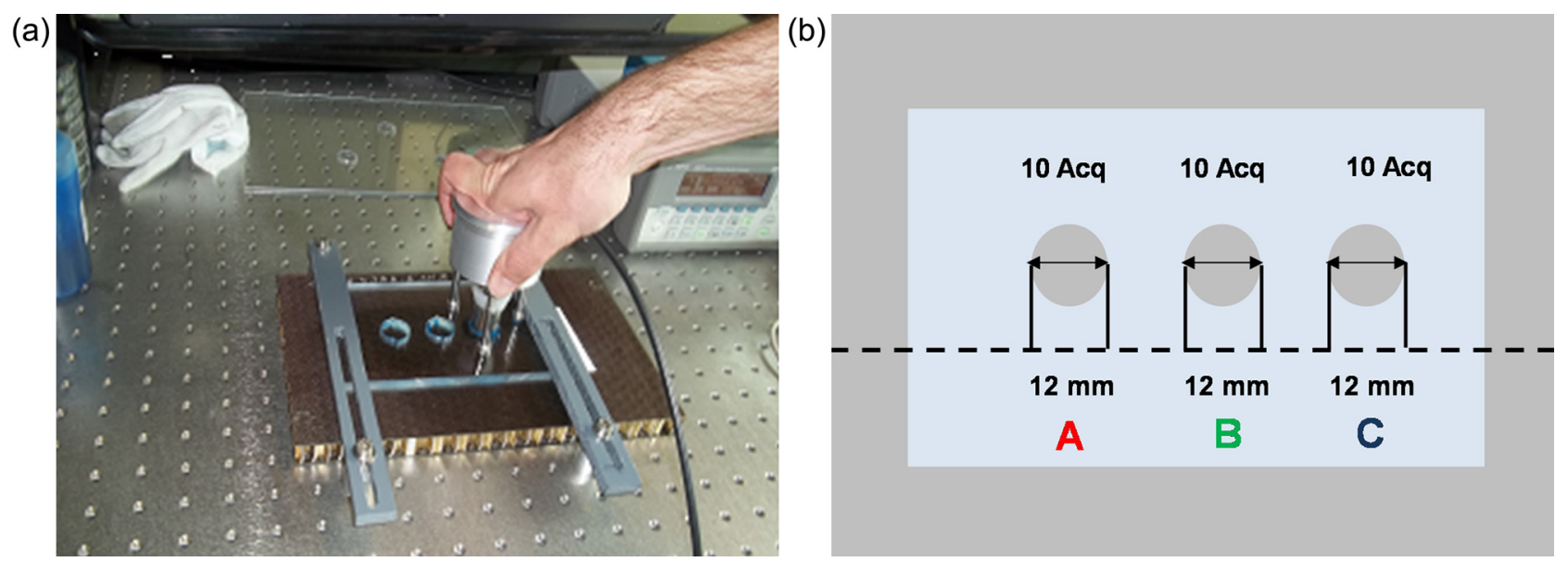

FIG. 13. (Color online) Electromechanical impedance measurement of the transducer coupled to a sandwich honeycomb plate: (a) experimental configuration, (b) scheme of the three acquisitions places $\mathrm{A}, \mathrm{B}$, and $\mathrm{C}$ on the material. 

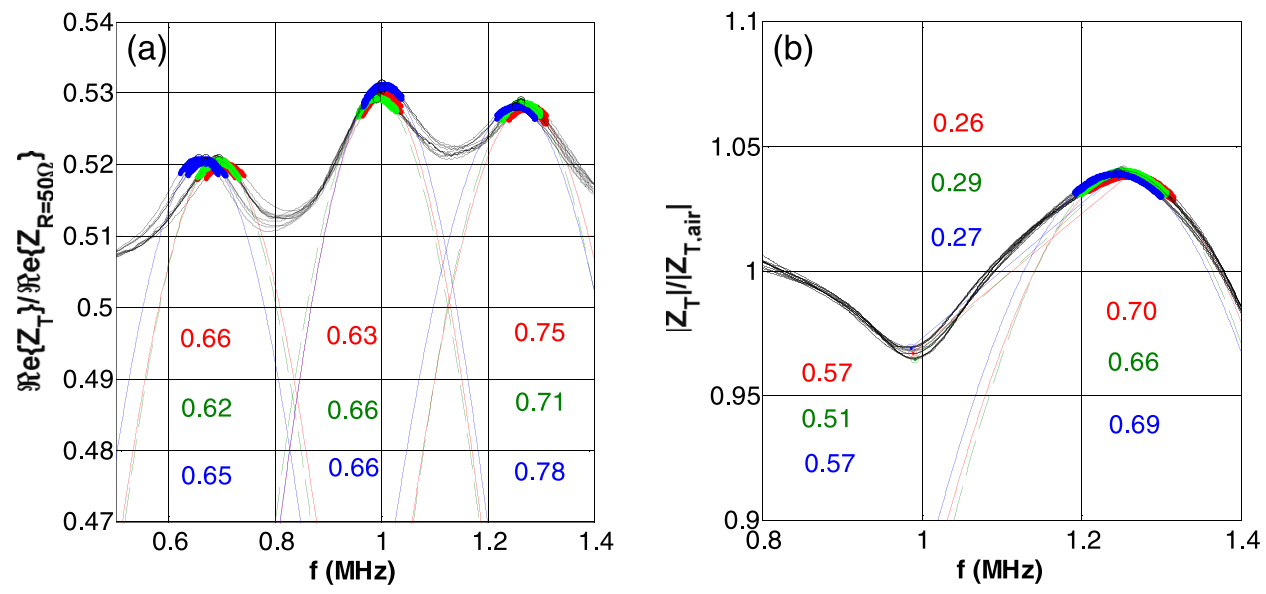

FIG. 14. (Color online) Measurement results on the reference plate: positions and Gaussian fits of peaks leading to the criteria values of (a) bandwidths c1, c2, c3 and (b) bandwidths c4, c5, and slope 66 .

durations of 200,800 , and $4000 \mathrm{~h}$. The two composite skins of each sample are cured at 2.5 and 7 bars, respectively. Due to the fabrication process, some imperfections in the sense of flatness are produced on one face of the skin. This kind of roughness due to the fiber tissue on one side of the plate is commonly called "telegraphing." The impedance measurements are made on the skin with a "telegraphing" (2.5 bars). As illustrated by Fig. 13(b), for a better characterization of these sandwich plates, three sets of ten measurements are made at points A, B, and C. Measurements are acquired by positioning the transducer in the cell center of the honeycomb. Regarding the measurement results, the values obtained at point $\mathrm{A}$ are mentioned in red, in green at point $\mathrm{B}$, and in red at point $\mathrm{C}$. We make this distinction to see the repeatability of measurements based on the heterogeneity of the plates, possibly due to the honeycomb cell size around $9.5 \mathrm{~mm}$.

\section{B. Results}

As for monolithic composite samples, the six criteria previously described are used. Figure 14(a) shows the results for the criteria c1-c3 ( $-3 \mathrm{~dB}$ widths) and Fig. 14(b) illustrates the criteria $\mathrm{c} 4-\mathrm{c} 6(-3 \mathrm{~dB}$ widths and slope) for the non-aged reference sandwich plate. Among the 10 measurements made at each point (A, B, and C), only the four closest ones are shown, and an average is made. The average value of the test is represented with the color defined before. In the following, we characterize the samples aged at $170^{\circ}$ and $200^{\circ} \mathrm{C}$ for periods ranging from 1 day $(24 \mathrm{~h})$ to 11 months $(8000 \mathrm{~h})$. Table III below summarizes the aging experienced on these sandwich plates denoted as "HT-D" of a given

TABLE III. Thermal aging of sandwich honeycomb plates at $170^{\circ} \mathrm{C}$ and $200^{\circ} \mathrm{C}$. The denomination of the thermal aging " $\mathrm{H} T-D$ " stands for " $\mathrm{H}$ " for honeycomb, $T\left({ }^{\circ} \mathrm{C}\right)$ for the temperature, and $D(\mathrm{~h})$ for the duration.

\begin{tabular}{lcc}
\hline \hline Duration $D(\mathrm{~h})$ & $T=170{ }^{\circ} \mathrm{C}$ & $T=200^{\circ} \mathrm{C}$ \\
\hline 24 & - & $\mathrm{H} 200-24$ \\
200 & $\mathrm{H} 170-200$ & $\mathrm{H} 200-200$ \\
800 & $\mathrm{H} 170-800$ & $\mathrm{H} 200-800$ \\
4000 & $\mathrm{H} 170-4000$ & $\mathrm{H} 200-4000$ \\
8000 & $\mathrm{H} 170-8000$ & - \\
\hline \hline
\end{tabular}

sample where " $\mathrm{H}$ " denotes honeycomb, $T\left({ }^{\circ} \mathrm{C}\right)$ represents the temperature of aging, and $D(\mathrm{~h})$ represents the duration of aging. As for the monolithic composite plates, Figs. 15(a) to 15(f) show the evolution of the normalized values $\mathrm{c} n / \mathrm{c} n_{\text {ref }}$ of each criterion $\mathrm{c} n(n=1,2, \ldots, 6)$ according to the duration of aging, respectively, and for the two temperatures of aging $170^{\circ} \mathrm{C}$ and $200^{\circ} \mathrm{C}$. The evolution of criteria $\mathrm{c} 1, \mathrm{c} 2, \mathrm{c} 4$, and c5 with the duration of aging in terms of time shows the expected progression of the thermal damage of the sandwich plates. The c6 criterion shows also a decreasing of the slope with the duration of aging. The same phenomenon was observable for the study of the monolithic plate (Fig. 12). For the $\mathrm{c} 3$ criterion, the evaluation of the ratio $\mathrm{c} 3 / \mathrm{c} 3$ ref is only carried out for the first three durations of aging at the two temperatures $170{ }^{\circ} \mathrm{C}$ and $200^{\circ} \mathrm{C}$. For the fourth duration of aging at the two temperatures (i.e., H170-8000 and


FIG. 15. (Color online) Comparison between aging at $T=170^{\circ} \mathrm{C}$ (blue squares) and $T=200^{\circ} \mathrm{C}$ (red circles) with the evolution of the criteria (a) $\mathrm{c} 1 / \mathrm{c} 1_{\text {ref }}$, (b) $\mathrm{c} 2 / \mathrm{c} 2_{\text {ref }}$, (c) $\mathrm{c} 3 / \mathrm{c} 3_{\text {ref }}$, (d) $\mathrm{c} 4 / \mathrm{c} 4_{\text {ref }}$, (e) $\mathrm{c} 5 / \mathrm{c} 5_{\text {ref }}$, (f) $\mathrm{c} 6 / \mathrm{c} 6_{\text {ref }}$, according to the aging duration for the sandwich honeycomb plates. 

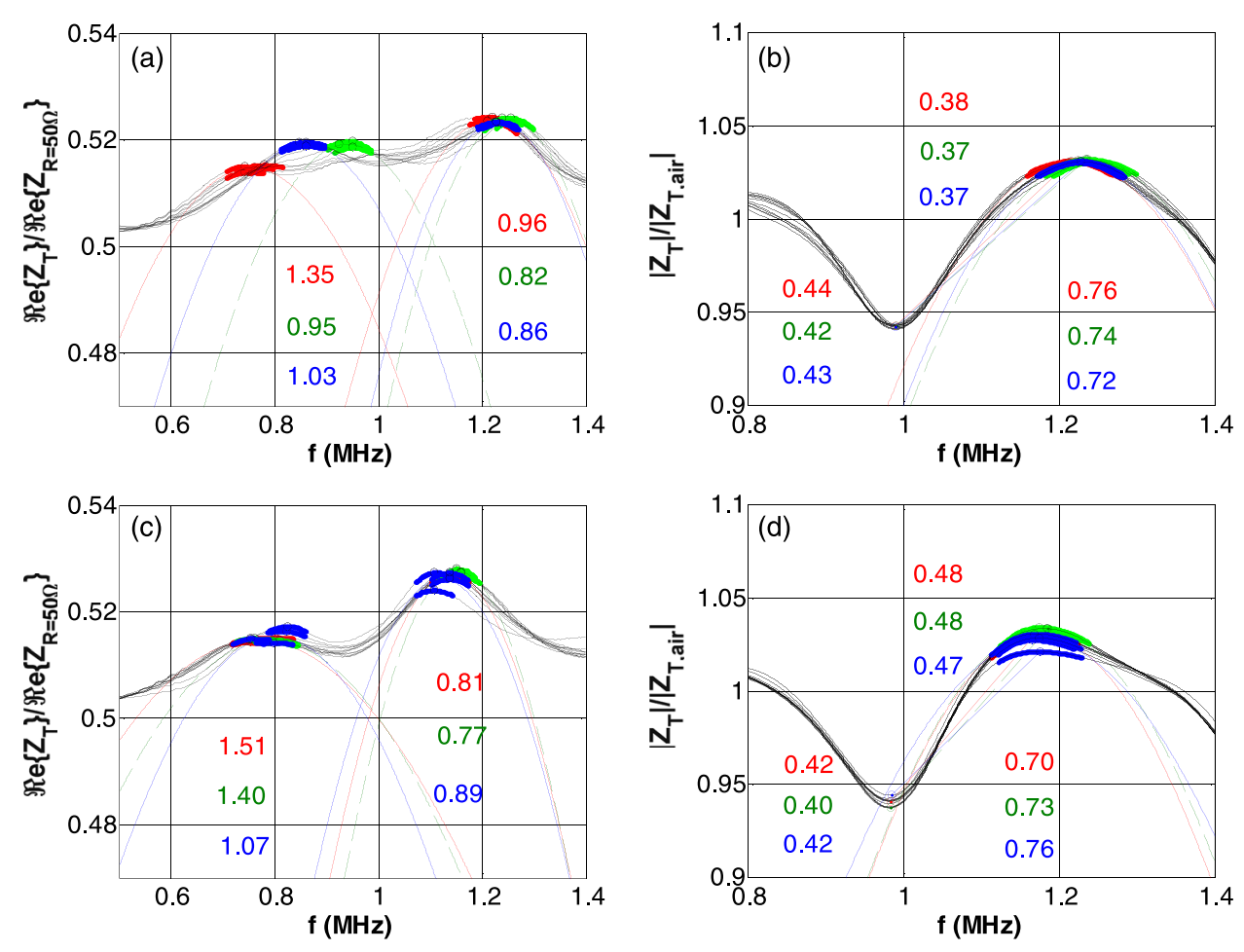

FIG. 16. (Color online) Measurement results on the aged plates: positions and Gaussian fits of peaks leading to the criteria values of (a), (c) bandwidths $\mathrm{c} 1$, c2, and (b), (d) bandwidths c4, c5, and slope c6, depending on the aging of the plates: (a), (b) H170-8000 and (c), (d) H200-4000.
H200-4000), the values of the c3 criterion are unquantifiable. This is due to the absence of a third peak of impedance at this more advanced thermal damage state. Figure 16 illustrates the advanced state of aging for the two sandwich honeycomb plates H170-8000 [Figs. 16(a) and 16(b)] and H200-4000 [Figs. 16(c) and 16(d)]. The third peak of impedance corresponding to $\mathrm{c} 3$ criterion disappears completely and the bandwidths of the other peaks increase considerably [Fig. 16(a) and 16(c)]. One can also see that the two factors of aging (i.e., temperature and time) have some considerable effects on the sandwich honeycomb plates.

\section{CONCLUSION}

In this study, an acoustical characterization composite plate was carried out by the measurement of the electromechanical impedance of a piezoelectric transducer. The inverse problem consisting in deducing relating the acoustical properties to the aging was lead extensively, depending on the aging conditions. An efficient technique based on Gaussian fits of the resonance characteristics led to new criteria successfully tested on thermal aging plates. This new method gives satisfactory results on monolithic plates. On much less ideal plates (cooked at low pressure, relatively high levels of porosity, telegraphing, etc.), the criteria change but the significant evolution of several criteria was highlighted according to thermal aging. Among the six criteria presented in this work, while tested on monolithic composite plates, three of them $\left(\mathrm{c} 1 / \mathrm{c} 1_{\text {ref }}\right.$, $\mathrm{c} 2 / \mathrm{c} 2_{\text {ref }}$, and $\mathrm{c} 3 / \mathrm{c} 3_{\text {ref }}$ ) offer both sensitivity from 1 for $500 \mathrm{~h}$ to more than 2 for $5000 \mathrm{~h}$ of aging, and robustness, i.e., continuous and monotonous variations. For the investigation led on sandwich honeycomb plates, the same criteria $\left(\mathrm{c} 1 / \mathrm{c} 1_{\text {ref }}\right.$, $\mathrm{c} 2 / \mathrm{c} 2_{\text {ref }}$, and $\mathrm{c} 3 / \mathrm{c} 3_{\text {ref }}$ ) show significant variations, but their sensitivity was reduced and their robustness was well-tested.
Particularly, the third criterion $\mathrm{c} 3 / \mathrm{c} 3$ ref broke down. The criteria $\left(\mathrm{c} 4 / \mathrm{c} 4_{\text {ref }}\right.$ and $\left.\mathrm{c} 5 / \mathrm{c} 55_{\text {ref }}\right)$ show some significant variations, but only for durations higher than $1000 \mathrm{~h}$, while the later c6/ c6 $6_{\text {ref }}$ stays flat, i.e., has no interest for this kind of material. The advantage of this technique is its easy implementation "under wing" in aeronautics. Where the usual techniques require plane surfaces without deformations, the method presented here can be complementary and can give an indication on the aging of materials.

\section{ACKNOWLEDGMENTS}

The authors are grateful to the CODAH for supporting this work and to André Baillard (Aircelle, Safran group) for his interest and fruitful discussions.

${ }^{1}$ Y. Deblock, P. Campistron, M. Lippert, and C. Bruneel, "Electrical characterization of plate piezoelectric transducers bonded to a finite substrate," J. Acoust. Soc. Am. 111(6), 2681-2685 (2002).

${ }^{2} \mathrm{C}$. Bois and C. Hochard, "Monitoring of laminated composites delamination based on electro-mechanical impedance measurement," J. Intell. Mater. Syst. Struct. 15, 59-67 (2004).

${ }^{3}$ C. Bois, P. Herzog, and C. Hochard, "Monitoring a delamination in a laminated composite beam using in-situ measurements and parametric identification," J. Sound Vib. 299(4-5), 786-805 (2007).

${ }^{4}$ I. Ammar-Khodja, C. Marais, C. Picard, M. Fois, and A. S. Goubet, "Comprehensive investigation on thermal degradation combined effects in aged woven carbon fibers/epoxy matrix composite laminates," in Proceedings of the 15th International Congress on Composite Materials (2005).

${ }^{5}$ Y. Gélébart, H. Duflo, and J. Duclos, "Air coupled lamb waves evaluation of the long-term thermo-oxidative ageing of carbon-epoxy plates," NDT\&E Int. 40(1), 29-34 (2007).

${ }^{6}$ I. Perrissin-Fabert and Y. Jayet, "Simulated and experimental study of the electric impedance of a piezoelectric element in a viscoelastic medium," Ultrasonics 32, 107-112 (1994).

${ }^{7}$ V. Giurgiutiu and C. A. Rogers, "Recent advancements in the electromechanical $(\mathrm{e} / \mathrm{m})$ impedance method for structural health monitoring and 
NDE," in SPIE's 5th Annual International Symposium on Smart Structures and Materials (1998).

${ }^{8}$ V. Giurgiutiu and A. Zagrai, "Damage detection in thin plates and aerospace structures with the electro-mechanical impedance method," Struct. Health Monit. 4(2), 99-118 (2005).

${ }^{9}$ H. Song, H. Lim, and H. Sohn, "Electromechanical impedance measurement from large structures using a dual piezoelectric transducer," J. Sound Vib. 332, 6580-6595 (2013).

${ }^{10}$ D. Wang, H. Song, and H. Zhu, "Numerical and experimental studies on admittances and correlation coefficient," Construct. Build. Mater. 49, 564-574 (2013).

${ }^{11}$ W. Yan, J. Cai, and W. Che, "An electro-mechanical impedance model of a cracked composite beam with adhesively bonded piezoelectric patches," J. Sound Vib. 330(2), 287-307 (2011).

${ }^{12}$ P. Selva, O. Cherrier, V. Budinger, F. Lachaud, and J. Morlier, "Smart monitoring of aeronautical composites plates based on electromechanical impedance measurements and artificial neural networks," Eng. Struct. 56, 794-804 (2013).

${ }^{13}$ W. P. Mason, Electromechanical Transducers and Wave Filters (Van Nostrand, New York, 1948).
${ }^{14}$ M. Redwood, "Transient performance of a piezoelectric transducer," J. Acoust. Soc. Am. 33(4), 527-536 (1961).

${ }^{15}$ D. Royer and E. Dieulesaint, Elastic Waves in Solids 2, translated by S. N. Lyle (Springer-Verlag, Berlin, 2000).

${ }^{16}$ V. G. M. Annamdas and C. K. Soh, "Embedded piezoelectric ceramic transducers in sandwiched beams," Smart Mater. Struct. 15(2), 538-549 (2006).

${ }^{17}$ O. Allix, "A composite damage meso-model for impact problems," Compos. Sci. Technol. 61, 2193-2205 (2001).

${ }^{18}$ H. J. Lim, M. K. Kim, H. Sohn, and C. Y. Park, "Impedance based damage detection under varying temperature and loading conditions," NDT\&E Int. 44(8), 740-750 (2011).

${ }^{19}$ N. Saint-Pierre, Y. Jayet, P. Guy, and J. Baboux, "Ultrasonic evaluation of dispersive polymers by the piezoelectric embedded element method: Modeling and experimental validation," Ultrasonics 36(6), 783-788 (1998).

${ }^{20}$ Y. Jayet, N. Saint-Pierre, J. Tatibouet, and D. Zellouf, "Monitoring the hydrolytic degradation of composites by a piezoelectric method," Ultrasonics 34(2-5), 397-400 (1996).

${ }^{21}$ J. Reddy, "On laminated composite plates with integrated sensors and actuators," Eng. Struct. 21(7), 568-593 (1999). 